\title{
Vacuum Decay Constraints on a Cosmological Scalar Field
}

\section{Citation}

Heyl, Jeremy S., and Abraham Loeb. 2002. "Vacuum Decay Constraints on a Cosmological Scalar Field." Physical Review Letters 88 (12). https://doi.org/10.1103/physrevlett.88.121302.

\section{Permanent link}

http://nrs.harvard.edu/urn-3:HUL.InstRepos:41417284

\section{Terms of Use}

This article was downloaded from Harvard University's DASH repository, and is made available under the terms and conditions applicable to Other Posted Material, as set forth at http:// nrs.harvard.edu/urn-3:HUL.InstRepos:dash.current.terms-of-use\#LAA

\section{Share Your Story}

The Harvard community has made this article openly available.

Please share how this access benefits you. Submit a story.

\section{Accessibility}




\title{
Vacuum Decay Constraints on a Cosmological Scalar Field
}

\author{
Jeremy S. Heyl \& Abraham Loeb \\ Harvard-Smithsonian Center for Astrophysics, MS-51, 60 Garden Street, Cambridge 02138
}

(November 5, 2018)

\begin{abstract}
If the potential of a scalar field $\phi$ which currently provides the "dark energy" of the universe, has a negative minimum $-M_{0}^{4}$, then quantum-mechanical fluctuations could nucleate a bubble of $\phi$ at a negative value of the potential. The bubble would expand at the speed of light. Given that no such bubble enveloped us in the past, we find that any minimum in $V(\phi)$ must be separated from the current $\phi$ value by more than $\min \left\{1.5 M_{0}, 0.21 M_{\mathrm{Pl}}\right\}$, where $M_{\mathrm{Pl}}$ is the Planck mass. We also show that vacuum decay renders a cyclic or ekpyrotic universe with $M_{0}^{4} \gtrsim 10^{-10} M_{\mathrm{Pl}}^{4}$, untenable.
\end{abstract}

PACS numbers: 98.80.Es, 98.80.Cq, 03.70.+k

Observations of Type Ia supernova [1] and microwave background anisotropies [2] indicate that the universe is currently dominated by "dark energy" with a negative pressure. One of the simplest realizations of such energy is in the form of a nearly massless cosmological scalar field $\phi$ ("quintessence") which is rolling down a shallow potential $V(\phi)$ and leading to an accelerated expansion of the universe at the current epoch. Can this potential take any arbitrary form for large deviations of $\phi$ from its present value $\langle\phi\rangle$ ? In this Letter we place interesting constraints on the shape of $V(\phi)$ by considering the possibility of vacuum decay. If $V(\phi)$ has a negative minimum, then a rare quantum-mechanical fluctuation in $\phi$ could nucleate a bubble inside of which the energy gained from $V(\phi)$ is larger than the energy invested in the gradients of $\phi$ on the bubble walls. Once nucleated, the walls of the bubble will propagate outwards similarly to a relativistic burning front and eventually envelope the entire volume within its future light cone, transforming $\phi$ to a lower energy state. A universe in which such nucleation events occur would become highly inhomogeneous and would evolve differently than expected based on the smooth semi-classical trajectory of $\phi$.

Bubble nucleation could have fatal consequences for some cosmological models. For example, it has recently been proposed [3] that a potential which reaches a minimum within a narrow and deep "pit" in Planck mass units $\left(M_{\mathrm{Pl}}=1.2 \times 10^{19} \mathrm{GeV}\right)$ can drive a cyclic universe with an infinite sequence of 'big bang'-'big crunch' cycles along its history. We will show that if the potential has a non-negligible slope in Planck mass units, then the vacuum in such a universe will inevitably become unstable and highly inhomogeneous during its big crunch. More generally, we will attempt to derive a condition for the stability of the vacuum in a cosmology with an arbitrary $V(\phi)$. We start by analysing the formation of a single bubble.

Bubble formation. We consider a cosmological scalar field with a general potential $V(\phi)$ that varies smoothly (without a barrier) towards a minimum value of $-M_{0}^{4}$ in a pit centered at $\phi=\phi_{\min }$ (see Fig. 1; we use units of $c=\hbar=1$ ). This generic shape has been suggested in the

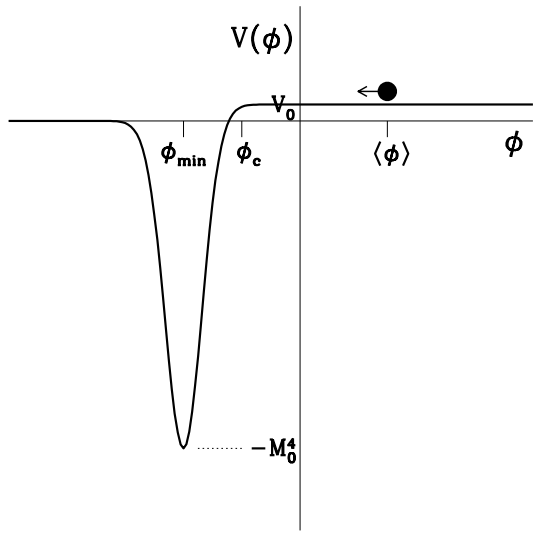

FIG. 1. The potential of a cosmological scalar field under consideration. The potential obtains a minimum of $-M_{0}^{4}$ at $\phi_{\min }$ which is much deeper than its current positive value $V_{0}$ at $\langle\phi\rangle$. This shape was inspired recently by string theory (see [3-5] and references therein).

context of a collision between two brane worlds which approach one another along an extra dimension (see [3$5]$ and references therein). The potential is relatively flat for $\phi>\phi_{\mathrm{c}}$ but declines rapidly for $\phi_{\min }<\phi<\phi_{\mathrm{c}}$.

Classically, the field is expected to reach the potential minimum throughout the universe in the future. The current potential value of $V_{0}$ at $\phi=\langle\phi\rangle$ (corresponding to a cosmological density parameter $\Omega_{\Lambda}=0.7$ ) is $\sim 10^{-123} M_{\mathrm{Pl}}^{4}$, while $M_{0}$ is expected to have a nonnegligible value in $M_{\mathrm{Pl}}$ units. Since $V_{0} \ll M_{0}^{4}$, we may ignore the small positive value of $V_{0}$ and assume that the potential is perfectly flat for $\phi>\phi_{\mathrm{c}}$. In order to treat the large derivative of $V(\phi)$ at $\phi<\phi_{\mathrm{c}}$ with the smallest number of free parameters, we approximate the potential as linear in this regime and consider

$$
V(\phi)=-M_{0}^{4} \frac{\phi-\phi_{\mathrm{c}}}{\phi_{\min }-\phi_{\mathrm{c}}} \Theta\left(\phi_{\mathrm{c}}-\phi\right),
$$

where $\Theta(x)$ is the Heaviside step function. For reasons that will become apparent below we are not concerned with the behavior of $V(\phi)$ at $\phi<\phi_{\min }$. 
Lee and Weinberg [8] calculated the probability of forming a bubble within which $\phi<\langle\phi\rangle$ for the potential in Eq. (1), neglecting the effects of gravity. We have verified that the positive contribution of the gravitational energy can be self-consistently neglected in their solution as long as 7 ( $\left(\langle\phi\rangle-\phi_{\mathrm{c}}\right) \ll \frac{3}{8 \sqrt{\pi}} M_{\mathrm{Pl}}$. We focus our discussion on this regime.

The field configuration that extremizes the Euclidean action for this potential, i.e. the "bounce", has the value of $\phi$ vary between $\phi=\langle\phi\rangle$ and $\phi=2 \phi_{\mathrm{c}}-\langle\phi\rangle$; therefore, if $\left(\langle\phi\rangle-\phi_{\mathrm{c}}\right)<\left(\phi_{\mathrm{c}}-\phi_{\min }\right)$, the "bounce" configuration is restricted to $\phi>\phi_{\min }$. Generically, $\left(\phi_{\mathrm{c}}-\phi_{\min }\right) \sim M_{\mathrm{Pl}}$ [3], and so the condition that gravity be unimportant is sufficient to ensure that the "bounce" does not go past the minimum of the potential.

The production rate per unit volume of bubbles is [8]

$$
\begin{aligned}
\lambda=\frac{4}{9} \pi^{2} A & \left(\langle\phi\rangle-\phi_{\mathrm{c}}\right)^{4} \times \\
& \exp \left\{-\frac{32 \pi^{2}}{3} \frac{\left(\langle\phi\rangle-\phi_{\mathrm{c}}\right)^{3}\left(\phi_{\mathrm{c}}-\phi_{\min }\right)}{M_{0}^{4}}\right\},
\end{aligned}
$$

where $A$ is a constant of order unity. Once formed the bubbles expand at the speed of light [7,8], so the typical time $t_{\text {tunnel }}$ after which the fraction $p$ of the space still remains outside of a bubble is [8],

$$
t_{\text {tunnel }}=\left[\frac{3}{\pi \lambda} \ln \frac{1}{p}\right]^{1 / 4} .
$$

If $t_{\text {tunnel }}$ is shorter than the classical, cosmological rolling time of the field $t_{\text {roll }}$, then the evolution of the field will be dominated by bubble formation.

While the field lies on the nearly flat part of the potential, $t_{\text {roll }} \sim\left(H_{0}\right)^{-1} \sim 10^{61} M_{\mathrm{Pl}}^{-1}$, where $H_{0}$ is the current Hubble constant. For $p=1 / e$ we find that $t_{\text {tunnel }}>t_{\text {roll }}$ if

$$
\left(\langle\phi\rangle-\phi_{\mathrm{c}}\right)^{3}\left(\phi_{\mathrm{c}}-\phi_{\min }\right)>5.4 M_{0}^{4} .
$$

where we have crudely substituted $\left(\langle\phi\rangle-\phi_{\mathrm{c}}\right) \sim M_{\mathrm{Pl}}$ in the pre-factor of the exponential in Eq. (2). The con-

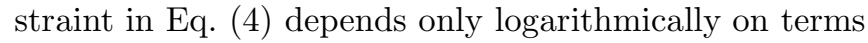
that appear outside the exponential; for example, if we take $t_{\text {roll }}$ to be $1 \mathrm{yr}$ instead of $10^{10}$ yr then the righthand-side of the inequality changes its value only slightly to $4.5 M_{0}^{4}$. As argued for standard inflation [10], if $t_{\text {tunnel }}<t_{\text {roll }}$ then the resulting universe will be highly inhomogeneous.

\footnotetext{
* Interestingly, even though the bubbles under consideration are not characterized by a thin wall, we find that the condition for neglecting gravity is similar to that obtained for potentials that satisfy the thin wall approximation but have a non-negligible wall thickness [7, g].
}

In conclusion, we find that the formation of a bubble inside the false vacuum of an evolving scalar field freezes out the quantum fluctuations of the field. During the current epoch of cosmic acceleration, the scale of the horizon is $\sim 61$ orders of magnitude greater than that of a Planck-scale bubble that could begin to grow. If the largest quantum fluctuation within the horizon is sufficiently large, a critical bubble will form and expand to contain its future light cone. The largest fluctuation within our horizon is typically a $\sim 30-\sigma$ event, i.e. it is astronomically rare, but if this fluctuation is large enough to create an expanding bubble, then the fluctuation is frozen out and renders the universe highly inhomogeneous.

Consequences today. Since the Earth has not been enveloped inside a bubble where the scalar field lies on the "cliff" of the potential and has a negative energy density, we find from Eq. (4) that today

$$
\left(\langle\phi\rangle-\phi_{\mathrm{c}}\right)>\min \left\{\frac{1.75 M_{0}^{4 / 3}}{\left(\phi_{\mathrm{c}}-\phi_{\min }\right)^{1 / 3}}, \frac{3}{8 \sqrt{\pi}} M_{\mathrm{Pl}}\right\} .
$$

This constrains the mean slope of the potential for $\phi>$ $\left[\langle\phi\rangle-3 /(8 \sqrt{\pi}) M_{\mathrm{pl}}\right]$ to be less than $0.002 M_{\mathrm{Pl}}^{3}$. It also implies that $\left(\langle\phi\rangle-\phi_{\min }\right)>\min \left\{1.5 M_{0}, 0.21 M_{\mathrm{Pl}}\right\}$.

Next we demonstrate the significance of our results in the context of specific cosmological models.

Implications for a cyclic or an ekpyrotic universe. Steinhardt \& Turok [3] have recently proposed a model for a cyclic universe based on a potential similar to that illustrated in Fig. 1; the universe in this model is currently dominated by an effective scalar field and is beginning to inflate. They argue that if the scalar field passes through its global minimum after $\sim 100 e$-foldings, then the universe could recollapse and bounce uniformly. After $\sim 100 e$-foldings, a typical Hubble volume would be completely devoid of matter or debris from the previous cycle; however, the quantum fluctuations in the scalar field just before the recollapse would be able to seed the density perturbations in the next cycle of cosmic evolution 11].

If we consider a simple harmonic potential for the scalar field at values $\phi>\phi_{\mathrm{c}}$ and take the density of the flat universe today to be $30 \%$ matter and $70 \%$ vacuum energy, we find through a numerical integration that the scalar field reaches the potential minimum after $\sim 92$ $e$-folds. Looking back in time from the moment of recollapse, the value of $\left|\langle\phi\rangle-\phi_{\mathrm{c}}\right|$ at the beginning of the final $e$-fold is $3 \%$ of $M_{\mathrm{Pl}}$. If the potential is too steep a bubble will form within the past light cone and will be able to envelope the entire Hubble volume before the scalar potential reaches its minimum. For the scalar field to roll rather than tunnel, the slope past the cliff is bounded by

$$
\frac{M_{0}^{4}}{\phi_{\mathrm{c}}-\phi_{\min }}<5 \times 10^{-6} M_{\mathrm{Pl}}^{3} \text {. }
$$


This limit cannot be evaded without increasing the rate of change of $\phi$, which would reduce the number of $e$-foldings before the field reaches the minimum. As $\langle\phi\rangle$ approaches the potential minimum, more bubbles could form and expand if the depth of the potential minimum is not too small. The formation of these bubbles destroys the homogeneity of the causally connected patch of the universe. In the cyclic universe the expansion will decelerate about one billion years before the potential reaches its minimum, because at this time the kinetic energy of the field begins to dominate the potential energy. Based on a numerical integration of the evolution equation for $\phi$, we find that expanding bubbles will form at this stage unless

$$
\frac{M_{0}^{4}}{\phi_{\mathrm{c}}-\phi_{\min }}<9 \times 10^{-11} M_{\mathrm{Pl}}^{3} .
$$

This constraint requires fine-tuning since the generic value of the slope lies in the Planck regime.

If condition (7) is not satisfied as the field approaches the cliff in the potential (where $d V / d \phi$ changes dramatically), expanding bubbles of negative energy density will begin to form and destroy the homogeneity of the collapse. The transition of the universe from an expanding phase to a contracting phase would not be uniform but rather proceed as a first-order phase transition with bubbles of contracting (anti-de Sitter) spacetime appearing in the expanding background. The walls of the internally contracting bubbles expand at the speed of light. Furthermore, because the interior of the bubble will deviate from spherical symmetry, possibly as a result of amalgamating a smaller bubble or inevitably from quantum fluctuations, the collapse within the bubble will be chaotic and singular. How these bubbles overlap and how chaotic their subsequent collapse is, will depend on the precise details of the phase transition. An orderly collapse and reexpansion cannot be achieved naturally in the context of the cyclic model. (For bubble dynamics in a rapidly inflating universe, see [10].) We note that in similarity to other tunneling problems, the exponentially-suppressed quantum-mechanical jump to a new, fully nonlinear configuration of $\phi$ may not be evident in any order of perturbation theory that was discussed previously in the literature [1].

Our conclusions apply also to the ekpyrotic universe [4,5 in which the big bang was preceded by a big crunch through a potential similar to that illustrated in Fig. 1 but with $V_{0}=0$. Since the small value of $V_{0}$ was ignored in our discussion, the above results apply to this case as well. In the ekpyrotic scenario, the universe exists for a sufficiently long time to smooth out inhomogeneities causally before collapsing in a big crunch and rebounding in a big bang. The constraints derived here apply also to this scenario since many bubbles of collapsing spacetime would form before the scalar field rolls down the potential classically and initiates the big crunch globally.
We have found that cosmological scenarios which require the universe to contract and rebound suffer from a first-order phase transition which necessarily destroys the homogeneity of the universe. Perhaps a sufficiently finely tuned potential for the scalar field which drives the evolution can avoid this first-order phase transition but it may not possess the other attractive features of the ekpyrotic and cyclic propositions. More generally, we have constrained the future classical evolution of the observed cosmological constant by appealing to the apparent lack of bubbles of collapsing spacetime in the universe today.

Acknowledgments. We thank Paul Steinhardt and Erick Weinberg for useful discussions. J.S.H. has been supported by the Chandra Postdoctoral Fellowship Award \# PF0-10015 issued by the Chandra X-ray Observatory Center, which is operated by the Smithsonian Astrophysical Observatory for and on behalf of NASA under contract NAS8-39073. This work has also been supported in part by grants of A.L. from NASA (NAG 5-7039, 5-7768), and NSF (AST-9900877, AST-0071019).

[1] A. Riess, et al., Astron. J., 116, 1009 (1998); S. Perlmutter, et al., Astrophys. J., 517, 565 (1999) .

[2] P. de Bernardis et al., Nature, 404, 955 (2000); S. Hanany, et al., Astrophys. J. Lett., 545, L5 (2000); B. Netterfield et al., astro-ph/0104460 (2001); A.T. Lee, et al. Astrophys. J., 561, L1 (2001); X. Wang, M. Tegmark, \& M. Zaldarriaga, astro-ph/0105091 (2001).

[3] P. J. Steinhardt \& N. Turok, hep-th/0111030 \& hepth/0111098 (2001).

[4] J. Khoury, B. A. Ovrut, N. Seiberg, P. J. Steinhardt, \& N. Turok, hep-th/0103239 \& hep-th/0108187 (2001).

[5] A. Linde, to appear in Proc. of PASCOS 2001, hepth/0107176 (2001)

[6] S. Coleman, Phys. Rev. D15, 2929 (1977).

[7] S. Coleman \& F. De Luccia, Phys. Rev. D21, 3305 (1980).

[8] K. Lee \& E. J. Weinberg, Nuc. Phys. B267, 181 (1986).

[9] K. Lee, Nuc. Phys. B, 282, 509 (1987).

[10] S. W. Hawking, I. G. Moss, \& J. M. Stewart, Phys. Rev. D26, 2681 (1982); A. H. Guth \& E. J. Weinberg, Nucl. Phys. B212, 321 (1983).

[11] J. Khoury, B. A. Ovrut, P. J. Steinhardt, \& N. Turok, hep-th/0109050 (2001). 